\title{
ESTILO ATRIBUCIONAL EN LENGUA Y RECHAZO ESCOLAR EN NIÑOS ESPAÑOLES
}

\author{
Mariola Giménez-Miralles \\ Universidad de Alicante \\ mgm141@gcloud.ua.es \\ Carolina Gonzálvez \\ Universidad de Alicante \\ carolina.gonzalvez@ua.es \\ María Pilar Aparicio-Flores \\ Universidad de Alicante \\ pilar.aparicio@ua.es
}

Recepción Artículo: 27 octubre 2021

Admisión Evaluación: 27 octubre 2021

Informe Evaluador 1: 28 octubre 2021

Informe Evaluador 2: 29 octubre 2021

Aprobación Publicación: 30 octubre 2021

\section{RESUMEN}

Los problemas de asistencia a la escuela son un tema candente dentro de la sociedad en la que nos encontramos ya que poseen un impacto significativo en el desarrollo del alumnado. El objetivo de este estudio consiste en comprobar si existen diferencias en las puntuaciones medias entre los sujetos con bajas y altas puntuaciones en rechazo escolar en función de las dimensiones de las atribuciones académicas en Lengua. Se administraron la Sydney Attribution Scale (SAS) y la School Refusal Assessment Scale-Revised (SRAS-R) a 732 estudiantes (52\% chicos) comprendidos entre 8 y 11 años $(M=9.71 ; S D=1.06)$. A partir de los resultados hallados, se confirma la hipótesis de inicio, según la cual se esperaba que el alumnado con altas puntuaciones en rechazo escolar obtuviera puntuaciones significativamente superiores en su atribución del fracaso a la falta de capacidad y esfuerzo. Esto ocurrió en aquellos estudiantes que basaban su rechazo a la escuela en evitar la afectividad negativa generada por determinadas situaciones escolares o escapar de situaciones de aversión social o evaluación. La actual situación ocasionada por la pandemia de la COVID-19, ha acentuado los problemas de la sociedad, y, sobre todo, del alumnado y todo lo que ello conlleva. Con el propósito de prevenir los problemas subyacentes es necesario conocer las necesidades de este alumnado y formar a los docentes para que sepan detectar estas dificultades a la hora de asistir a la escuela y estar preparados para ayudar al alumnado en su proceso de enseñanza-aprendizaje.

Palabras clave: atribuciones académicas; lengua; éxito; fracaso; rechazo escolar; problemas de asistencia escolar 


\title{
ESTILO ATRIBUCIONAL EN LENGUA Y RECHAZO ESCOLAR EN NIÑOS ESPAÑOLES
}

\begin{abstract}
Differences between success and failure in academic self-attributions in Language and Literature. School attendance problems are a hotly debated topic in our society as they have a major impact in the students' development. The objective of this research consisted of assessing the differences in the average scores between the individuals who achieved low and high scores in school refusal behavior related to academic self-attributions in Language. The Sydney Attribution Scale (SAS) and the School Refusal Assessment-Scale (SRAS-R) were administered to 732 students (52\% boys) aged 8 to $11(M=9.71 ; S D=1.06)$. From the results found, the hypothesis is confirmed, since it was expected that students with high scores in school refusal behavior obtained significantly higher scores on attributions of failure to ability and effort. This occurred in those students who based their rejection of school on avoiding the negative affectivity generated by certain school situations or escaping from situations of social aversion or evaluation. The current situation caused by the COVID-19 pandemic has accentuated the problems of society, and, above all, of the students and all that this entails. In order to prevent underlying problems, it is necessary to know the needs of these students and train teachers so that they know how to detect these difficulties when attending school and be prepared to help students in their teaching-learning process.
\end{abstract}

Keywords: academic self-attributions; language; success; failure; school refusal; school attendance problems.

\section{INTRODUCCIÓN}

La preocupación por temas como los problemas de asistencia en la escuela ha aumentado en los últimos años, debido a los efectos que provocan en el desarrollo social, emocional y educativo de los niños y jóvenes (Devenney y 0'Toole, 2021; Espada, et. al., 2012; Méndez, et. al., 2008). No asistir a la escuela se considera un incumplimiento del derecho básico a la educación (Donat et al., 2018), el cual se reconoce por primera vez en 1948 en la Declaración Universal de los Derechos Humanos ("el derecho a la educación con objecto del pleno desarrollo de la personalidad humana y el fortalecimiento del respeto a los derechos humanos y a las libertades fundamentales favoreciendo la comprensión, tolerancia y la amistad entre todas las naciones y todos los grupos étnicos y religiosos") (Ugarte, 2003).

Estudios recientes han identificado perfiles o subgrupos de estudiantes que comparten características relacionadas con problemas de asistencia escolar (García-Fernández, 2010; García-Fernández, 2013; Fornander y Kearney, 2020; Kearney et al., 2021). Estos estudios se basan en el modelo funcional, el cual incluye cuatro posibles causas para el rechazo del alumno a la escuela. Estas condiciones son: (1) Evitar la afectividad negativa que provocan los estímulos o situaciones relacionadas con el ámbito escolar. Los estudiantes con altas puntuaciones en este factor se asocian con el trastorno de ansiedad generalizada, súplicas constantes y quejas somáticas con el propósito de no ir a la escuela; (2) Escapar de la aversión social o situaciones de evaluación. Los estudiantes con altas puntuaciones en este factor se han vinculado al trastorno de ansiedad generalizada y ansiedad social, mostrando comportamientos retraídos; (3) Búsqueda de atención de otras personas significativas. Los estudiantes con puntuaciones altas en este factor correlacionan de manera positiva con el trastorno de ansiedad por separación y el trastorno negativista desafiante; y (4) Búsqueda de refuerzos tangibles fuera del ámbito escolar. Los estudiantes con altas puntuaciones en esta dimensión se han asociado con el trastorno negativista desafiante. En las primeras dos condiciones, el rechazo se mantiene por reforzamiento negativo, por ejemplo, vencer el pánico escénico en una exposición oral, mientras que en las dos últimas, se mantiene por el reforzamiento positivo, por ejemplo, quedarse en casa utilizando redes sociales.

En el estudio de los problemas de asistencia a la escuela además de preocupar su relación con distintos trastornos psicológicos preocupa su impacto sobre el rendimiento académico. En este sentido, resulta necesario analizar la relación existente entre el rechazo escolar y las atribuciones académicas. Éstas se presentan a partir de la teoría de la atribución causal de Weiner (1986) y se definen como percepciones descriptivas que el individuo presenta considerando que la atribución es un proceso de tres niveles en el que, primeramente, el comportamiento 
se analiza; en segundo lugar, se determina si el comportamiento es intencional; y finalmente, se asignan causas internas 0 externas a dicho comportamiento. Existen tres factores para percibir el éxito y fracaso, basados en el control del locus, la estabilidad y la controlabilidad: (1) causas internas o externas, dependiendo de la localización de la causa; (2) causas estables o inestables, de acuerdo con la perseverancia e inconsistencia de la causa; y (2) causas controlables e incontrolables tomando como referencia el grado de control de los individuos para adaptarse a las consecuencias (Martínez-Monteagudo, 2019; Weiner y Graham, 1990). Los estudiantes tienden a atribuir sus éxitos y fracasos a causas internas, como la capacidad, o falta de ella; y a causas externas, como la dificultad de una tarea 0 la carencia de suerte (Weiner, 1992). Aquel alumnado con puntuaciones altas de autoestima y éxito escolar atribuyen sus éxitos a factores internos, estables e incontrolables (como la capacidad), mientras que atribuyen sus fracasos a factores internos, inestables y controlables (por ejemplo, el esfuerzo), y a factores externos, estables e incontrolables como la dificultad de la tarea a realizar (Genç, 2016).

La relación entre el rechazo escolar y las atribuciones académicas se ha estudiado de manera limitada. Tan sólo se ha encontrado un par de investigaciones al respecto que revelan la necesidad de seguir ampliando la investigación en este campo. En cuanto al área de Matemáticas, Gonzálvez et al. (2018) identificaron en niños españoles los estudiantes con altas puntuaciones en rechazo escolar por reforzamiento negativo atribuían más sus fracasos a la falta de capacidad y esfuerzo, mientras que los estudiantes que justificaban su rechazo a la escuela en el cuarto factor de la SRAS-R (evitar ir a la escuela para obtener refuerzos tangibles externos a la escuela) atribuían más sus éxitos a la capacidad. Sin embargo, no se dispone de datos acerca de las puntuaciones medias de los estudiantes con rechazo escolar en función de su estilo atribucional en Lenguaje. En el área de Lengua tan sólo encontramos los recientes hallazgos de Gonzálvez et al. (2021) que tras identificar cuatro perfiles o grupos de estudiantes con rechazo escolar señalaron que aquellos estudiantes con altas puntuaciones en los tres primeros factores de la SRAS-R (perfil mixto de rechazo escolar) estos revelaron puntuaciones medias más altas en un estilo de autoatribución académico en el que asocian sus fracasos a la falta de habilidad y esfuerzo.

\section{OBJETIVOS DE LA INVESTIGACIÓN}

Este trabajo pretende comprobar si existen diferencias en las puntuaciones medias entre los sujetos con bajas y altas puntuaciones en rechazo escolar en función de las dimensiones de las atribuciones académicas en Lengua. Las atribuciones académicas evaluadas se presentan agrupadas según hagan referencia al éxito (capacidad, esfuerzo o causas externas) o fracaso (capacidad, esfuerzo o causas externas).

Tras efectuar el análisis de la literatura previa, se espera que el alumnado con altas puntuaciones en rechazo escolar para los dos primeros factores de la SRAS-R (1. Evitar la afectividad negativa que provocan los estímulos 0 situaciones relacionadas con el ámbito escolar y 2. Escapar de la aversión social o situaciones de evaluación) obtenga puntuaciones significativamente superiores en su atribución del fracaso a la falta de capacidad y esfuerzo (Gonzálvez et al., 2018, 2021; Sanmartín et al., 2016).

\section{MUESTRA Y/O PARTICIPANTES}

En este estudio participaron 732 niños españoles cuya edad osciló entre los 8 y 11 años ( $M=9.71$; $S D=$ 1.06). Previa a su participación en la investigación se informó a las familias del objetivo de la investigación solicitando su consentimiento informado por escrito.

\section{METODOLOGÍA E INSTRUMENTOS UTILIZADOS}

Con el objetivo de determinar la existencia de diferencias estadísticamente significativas entre las puntuaciones medias entre las variables de rechazo escolar y estilo atribucional académico en Lengua, se aplicó la prueba $t$ de Student, para la cual se dicotomizaron los datos del rechazo escolar. Se consideraron altas puntuaciones en rechazo escolar las puntuaciones iguales 0 superiores al percentil 75 y se consideraron bajas aquellas iguales 0 inferiores al percentil 25. Además, se calculó el índice d (diferencia media tipificada) propuesto por Cohen (1988) para establecer la magnitud del efecto de las diferencias encontradas considerando que una magnitud es pequeña 


\section{ESTILO ATRIBUCIONAL EN LENGUA Y RECHAZO ESCOLAR EN NIÑOS ESPAÑOLES}

si se encuentra entre .20 y .49, es moderada entre .50 y .79 , y es grande si es superior a .80. El programa estadístico empleado en esta investigación ha sido el SPSS 24.

Los instrumentos utilizados en esta investigación fueron dos y se presentan a continuación:

Sydney Attribution Scale (SAS) (Marsh et al., 1984) consta de 24 situaciones hipotéticas que deben ser consideradas por los participantes. Utilizando una escala de tipo Likert de 5 puntos ( $1=$ falso; $5=$ verdad), evalúa la causa percibida por los estudiantes cuando se refieren a sus éxitos y fracasos. De este modo, en la presente investigación sólo se utilizaron aquellas situaciones referidas al área de Lengua. Los coeficientes de consistencia interna en este trabajo fueron de 82 para Éxito/Capacidad, de .74 para Éxito/Esfuerzo, de .72 para Éxito/Causas Externas, de .77 para Fracaso/Capacidad, de .74 para Fracaso/Esfuerzo y de 69 para Fracaso/Causas Externas.

School Refusal Assessment Scale-Revised (SRAS-R) (Kearney, 2002) es un instrumento de autoevaluación formado por 24 items usando una escala con 7 puntos de respuesta ( $0=$ nunca; $6=$ siempre). Evalúa las cuatro condiciones funcionales para que permanezca un comportamiento de rechazo escolar: (1) Evitar la afectividad negativa que provocan los estímulos o situaciones relacionadas con el ámbito escolar; (2) Escapar de la aversión social o situaciones de evaluación; (3) Búsqueda de atención de otras personas significativas; y (4) Búsqueda de refuerzos tangibles fuera del ámbito escolar. En el presente estudio se utilizó la versión española del SRAS-R (Gonzálvez et al., 2016) con 18 ítems y manteniendo los cuatro factores previamente mencionados. Los coeficientes de consistencia interna fueron: .74 (ANA), ,86 (ESE), .75 (PAS) and .71 (SAS) para este estudio.

\section{RESULTADOS ALCANZADOS}

\section{Diferencias en las atribuciones académicas y rechazo escolar (Factor I)}

La tabla 1 presenta las diferencias en atribuciones en Lengua en función de las altas y bajas puntuaciones en rechazo escolar del Factor I (Evitar la afectividad negativa que provocan los estímulos o situaciones relacionadas con el ámbito escolar). Los estudiantes con bajas puntuaciones en rechazo escolar para el Factor I obtuvieron puntuaciones medias significativamente más altas a la hora de atribuir sus éxitos académicos a la capacidad, el esfuerzo o variables externas, que sus iguales con altas puntuaciones en rechazo escolar. Sin embargo, Ios estudiantes con altas puntuaciones en rechazo escolar para este factor obtuvieron puntuaciones medias significativamente más altas que sus iguales con bajas puntuaciones en las atribuciones académicas al fracaso debido a la falta de capacidad y esfuerzo. En cuanto a las atribuciones académicas del fracaso debido a causas externas, fueron los estudiantes con bajas puntuaciones en rechazo escolar para el este factor los que revelaron puntuaciones medias más altas. La magnitud de estas diferencias oscila entre 29 y .58, encontrando diferencias pequeñas para las atribuciones del éxito a la capacidad (.45) y esfuerzo (.37), diferencias del fracaso debido a la falta de esfuerzo (-.29) y falta de capacidad (-.41). Además, existen diferencias moderadas para las atribuciones del éxito a causas externas (.58) y atribuciones del fracaso a causas externas (.54). No se encontraron diferencias de tamaño grandes. 
Tabla 1. Diferencias en atribuciones en lengua en función de las altas y bajas puntuaciones en rechazo escolar (Factor I)

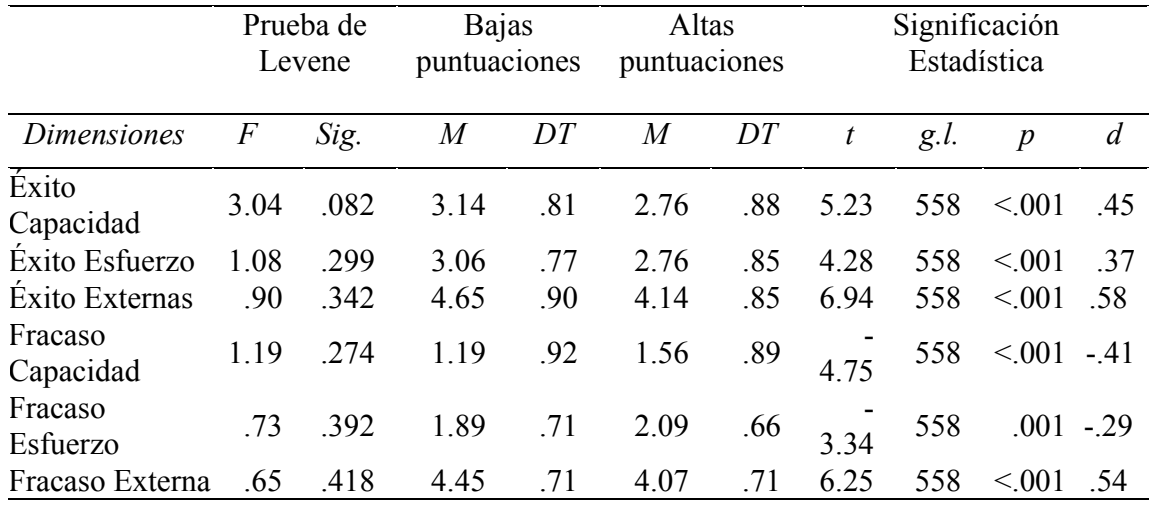

\section{Diferencias en las atribuciones académicas y rechazo escolar (Factor II)}

La tabla 2 muestra las diferencias en atribuciones en Lengua en función de las altas y bajas puntuaciones en rechazo escolar del Factor II (Escapar de la aversión social o situaciones de evaluación). Los estudiantes con bajas puntuaciones en rechazo escolar para el Factor II obtuvieron puntuaciones medias más altas a la hora de atribuir sus éxitos académicos a la capacidad y variables externas, y también a la hora de atribuir sus fracasos a causas externas, si los comparamos con sus iguales con altas puntuaciones en rechazo escolar. A su vez, mostraron diferencias significativamente más altas en la atribución del éxito a su esfuerzo. № obstante, los estudiantes con altas puntuaciones en rechazo escolar para este factor obtuvieron puntuaciones medias más altas que sus iguales con bajas puntuaciones en las atribuciones académicas al fracaso debido a la falta de capacidad y esfuerZ0. La magnitud de estas diferencias oscila entre .22 y .46, hallando diferencias pequeñas para las atribuciones del éxito a la capacidad (.39), esfuerzo (.22) y causas externas (.46), y para las atribuciones del fracaso a falta de capacidad (-.45), falta de esfuerzo (-.18), y causas externas (.36). No se hallaron diferencias de tamaño moderado 0 grande.

Tabla 2. Diferencias en atribuciones en lengua en función de las altas y bajas puntuaciones en rechazo escolar (Factor II)

\begin{tabular}{|c|c|c|c|c|c|c|c|c|c|c|}
\hline \multirow[b]{2}{*}{ Dimensiones } & \multicolumn{2}{|c|}{$\begin{array}{l}\text { Prueba de } \\
\text { Levene }\end{array}$} & \multicolumn{2}{|c|}{$\begin{array}{c}\text { Bajas } \\
\text { puntuaciones }\end{array}$} & \multicolumn{2}{|c|}{$\begin{array}{c}\text { Altas } \\
\text { puntuaciones }\end{array}$} & \multicolumn{4}{|c|}{$\begin{array}{c}\text { Significación } \\
\text { Estadística }\end{array}$} \\
\hline & $F$ & Sig. & $M$ & $D T$ & $M$ & $D T$ & $t$ & g.l. & $p$ & $d$ \\
\hline Éxito & 3.62 & .057 & 3.07 & .80 & 2.74 & .90 & 4.87 & 613 & $<.001$ & .39 \\
\hline Éxito Esfuerzo & .07 & .779 & 2.94 & .81 & 2.76 & .84 & 2.67 & 613 & .008 & .22 \\
\hline Éxito Externas & .46 & .496 & 4.54 & .87 & 4.14 & .86 & 5.72 & 613 & $<.001$ & .46 \\
\hline $\begin{array}{l}\text { Fracaso } \\
\text { Capacidad }\end{array}$ & .05 & .807 & 1.18 & .88 & 1.58 & .89 & -5.55 & 613 & $<.001$ & -.45 \\
\hline Fracaso Esfuerzo & .25 & .611 & 1.92 & .70 & 2.04 & .66 & -1.99 & 613 & .046 & -.18 \\
\hline Fracaso Externa & .57 & .448 & 4.34 & .73 & 4.08 & .71 & 4.40 & 613 & $<.001$ & .36 \\
\hline
\end{tabular}




\section{ESTILO ATRIBUCIONAL EN LENGUA Y RECHAZO ESCOLAR EN NIÑOS ESPAÑOLES}

\section{Diferencias en las atribuciones académicas y rechazo escolar (Factor III)}

La tabla 3 exhibe las diferencias en atribuciones en lengua en función de las altas y bajas puntuaciones en rechazo escolar del Factor III (Búsqueda de atención de otras personas significativas). El alumnado con bajas puntuaciones en rechazo escolar para el Factor III obtuvo puntuaciones más altas a la hora de atribuir sus éxitos a causas externas y a la hora de atribuir sus fracasos a causas externas, obteniendo en estas dimensiones diferencias significativas A pesar de este hecho, el alumnado con altas puntuaciones en rechazo escolar para este factor obtuvo diferencias significativas en las atribuciones del fracaso a la falta de capacidad. Para el resto de las comparaciones en este factor, entre los dos grupos para las distintas dimensiones del SAS, no se hallaron diferencias estadísticamente significativas. La magnitud de estas diferencias varía entre .18 y .34, encontrando únicamente diferencias pequeñas para las atribuciones del éxito a causas externas (.18), atribuciones del fracaso a la falta de capacidad (-.26) y, a causas externas (.34).

Tabla 3. Diferencias en atribuciones en lengua en función de las altas y bajas puntuaciones en rechazo escolar (Factor III)

\begin{tabular}{cccccccccccc}
\hline & $\begin{array}{c}\text { Prueba de } \\
\text { Levene }\end{array}$ & \multicolumn{3}{c}{$\begin{array}{c}\text { Bajas } \\
\text { puntuaciones }\end{array}$} & $\begin{array}{c}\text { Altas } \\
\text { puntuaciones }\end{array}$ & \multicolumn{4}{c}{$\begin{array}{c}\text { Significación } \\
\text { Estadística }\end{array}$} \\
\hline Dimensiones & $F$ & Sig. & $M$ & $D T$ & $M$ & $D T$ & $t$ & $g . l$. & $p$ & $d$ \\
\hline Éxito Capacidad & 2.51 & .114 & 2.95 & .82 & 2.84 & .91 & 1.36 & 554 & .173 & - \\
Éxito Esfuerzo & 3.37 & .067 & 2.84 & .78 & 2.87 & .86 & -.49 & 554 & .619 & - \\
Éxito Externas & .20 & .651 & 4.42 & .88 & 4.26 & .89 & 2.19 & 554 & .029 & .18 \\
$\quad$ Fracaso & .68 & .409 & 1.23 & .88 & 1.46 & .91 & -5 & 554 & .003 & -.26 \\
$\quad$ Capacidad & & & & & & & & \\
Fracaso Esfuerzo & .18 & .664 & 2.01 & .70 & 1.98 & .71 & .39 & 554 & .692 & - \\
Fracaso Externa & 1.26 & .261 & 4.34 & .67 & 4.10 & .75 & 3.92 & 554 & $<.001$ & .34 \\
\hline
\end{tabular}

\section{Diferencias en las atribuciones académicas y rechazo escolar (Factor IV)}

La tabla 4 muestra las diferencias en atribuciones en Lengua en función de las altas y bajas puntuaciones en rechazo escolar del Factor IV (Búsqueda de refuerzos tangibles fuera del ámbito escolar). El alumnado con bajas puntuaciones en rechazo escolar para el Factor IV obtuvo puntuaciones medias significativamente más altas con respecto a sus iguales con altas puntuaciones, cuando atribuyen sus fracasos a causas externas. Del mismo modo, el alumnado con altas puntuaciones en rechazo escolar presenta puntuaciones significativamente más altas a la hora de atribuir sus éxitos a la capacidad y al esfuerzo. La magnitud de estas diferencias oscila entre .20 y .39, encontrando diferencias pequeñas para las atribuciones del fracaso a causas externas (.20), atribuciones del éxito al esfuerzo (-.34) y capacidad (-.39). En las demás dimensiones no se encontraron diferencias significativas. 
Tabla 4. Diferencias en atribuciones en lengua en función de las altas y bajas puntuaciones en rechazo escolar (Factor IV)

\begin{tabular}{|c|c|c|c|c|c|c|c|c|c|c|}
\hline \multirow[b]{2}{*}{ Dimensiones } & \multicolumn{2}{|c|}{$\begin{array}{l}\text { Prueba de } \\
\text { Levene }\end{array}$} & \multicolumn{2}{|c|}{$\begin{array}{c}\text { Bajas } \\
\text { puntuaciones }\end{array}$} & \multicolumn{2}{|c|}{$\begin{array}{c}\text { Altas } \\
\text { puntuaciones }\end{array}$} & \multicolumn{4}{|c|}{$\begin{array}{c}\text { Significación } \\
\text { Estadística }\end{array}$} \\
\hline & $F$ & Sig. & $M$ & $D T$ & $M$ & $D T$ & $t$ & g.l. & $p$ & $d$ \\
\hline Éxito Capacidad & 5.46 & .020 & 2.74 & .87 & 3.06 & .77 & -4.70 & 563.9 & $<.001$ & -.39 \\
\hline Éxito Esfuerzo & 1.39 & .237 & 2.72 & .81 & 2.99 & .76 & -4.17 & 615 & $<.001$ & -.34 \\
\hline Éxito Externas & 2.67 & .102 & 4.31 & .86 & 4.40 & .89 & -1.29 & 615 & .195 & - \\
\hline $\begin{array}{l}\text { Fracaso } \\
\text { Capacidad }\end{array}$ & .81 & .367 & 1.35 & .84 & 1.32 & .87 & .33 & 615 & .739 & - \\
\hline Fracaso Esfuerzo & .46 & .494 & 1.94 & .68 & 2.02 & .65 & -1.37 & 615 & .171 & - \\
\hline Fracaso Externa & .66 & .416 & 4.29 & .67 & 4.15 & .72 & 2.39 & 615 & .017 & .20 \\
\hline
\end{tabular}

\section{DISCUSIÓN}

El objetivo del presente estudio era comprobar si existen diferencias en las puntuaciones medias entre los sujetos con bajas y altas puntuaciones en rechazo escolar en función de las dimensiones de las atribuciones académicas en Lengua, teniendo en cuenta que las atribuciones académicas evaluadas se presentan agrupadas según hagan referencia al éxito (capacidad, esfuerzo o causas externas) o fracaso (capacidad, esfuerzo causas externas).

A partir de los resultados hallados, se confirma la hipótesis de inicio, según la cual se esperaba que el alumnado con altas puntuaciones en rechazo escolar para los dos primeros factores de la SRAS-R obtuviera puntuaciones significativamente superiores en su atribución del fracaso a la capacidad y esfuerzo. Al igual que en el trabajo previo que había analizado la relación entre estas variables en el área de Matemáticas (Gonzálvez et al., 2018), nuestros hallazgos en el área de Lengua revelan que son los estudiantes con altas puntuaciones en rechazo escolar para los dos primeros factores de la SRAS-R los que atribuyen más sus fracasos a la falta de capacidad y esfuerzo. Por el contrario, el alumnado que justifica su rechazo a la escuela en la búsqueda refuerzos tangibles fuera del ámbito escolar, más asociado con alumnado de edades avanzadas que suele presentar absentismo escolar y que prefiere quedarse en casa para realizar actividades más recreativas, las presenta en su atribución del éxito a la capacidad y el esfuerzo. A pesar de que el estilo atribucional sea más adaptativo para este último grupo de estudiantes con rechazo escolar sería interesante analizar qué otras variables repercuten sobre el rendimiento académico del alumnado con el fin de esclarecer su relación.

Sería recomendable tener en consideración para futuras investigaciones las limitaciones encontradas en el presente estudio. Por un lado, sería necesaria una muestra más amplia y diversa teniendo en cuenta otros factores como el país de origen, la nacionalidad, el estatus socioeconómico de las familias, la salud o datos psicológicos del alumnado. A pesar de estas limitaciones, los resultados de la presente investigación pueden ser útiles como contribución al estudio de atribuciones académicas y su relación con el rechazo escolar.

\section{CONCLUSIONES}

La actual situación ocasionada por la pandemia de la COVID-19, ha acentuado los problemas de la sociedad, y, sobre todo, del alumnado y todo lo que ello conlleva. Los problemas de asistencia escolar son un tema recurrente hoy en día entre los que nos dedicamos al mundo de la Educación, Pedagogía, Psicología, etc. (Pérez et al., 2021). Con el propósito de prevenir los problemas subyacentes es necesario formar a los docentes que participan de la vida escolar del alumnado a que detecten estas dificultades y puedan participar de programas de técnicas de estudio, además de cursos de cuidado personal y ansiedad con su alumnado, ya que estas tienen una incidencia directa en la mejora del aprendizaje y su aplicación suele ser sencilla y eficaz (Guillen, 2020). La 


\section{ESTILO ATRIBUCIONAL EN LENGUA Y RECHAZO ESCOLAR EN NIÑOS ESPAÑOLES}

escuela tiene el deber de ser una constructora permanente de escenarios para el bienestar del estudiante, motivando la asistencia diaria de niños y jóvenes a los centros educativos (Vega, 2021).

\section{FINANCIACIÓN}

Esta investigación ha sido financiada por el Ministerio de Ciencia, Innovación y Universidades, y Fondos FEDER (RTI2018-098197-B-I00) otorgado a José M. García-Fernández y el Proyecto GV/2019/075 otorgado a Carolina Gonzálvez.

\section{REFERENCIAS BIBLIOGRÁFICAS}

Cohen, J. (1988). Statistical power analysis for the behavioral sciences. Hillsdale, NJ Lawrence Erlbaum Associates, Publishers.

Devenney, R., \& O'Toole, C. (2021). What kind of education system are we offering: the views of education professionals on school refusal. International Journal of Educational Psychology, 10(1), 27-47. https://doi.org/10.17583/ijep.2021.7304

Donat, M., Gallschütz, C., \& Dalbert, C. (2018). The relation between students' justice experiences and their school refusal behavior. Social psychology of education, 21(2), 447-475. https://doi.org/10.1007/s11218017-9423-9

Espada, J.P., Griffin, K. W., Pereira, J.R., Orgilés, M. y Garcia-Fernandez, J.M. (2012). Component Analysis of a School-Based Substance Use Prevention Program in Spain: Contributions of Problem Solving and Social Skills Training Content. Prevention Science, 13, 86-95. D0I: 10.1007/S11121-011-0249-Y

Fornander, M.J., \& Kearney, C.A. (2020). Internalizing symptoms as predictors of school absenteeism severity at multiple levels: Ensemble and classification and regression tree analysis. Frontiers in psychology, 10, 3079. https://doi.org/10.3389/fpsyg.2019.03079

Garcia-Fernandez, J. M., Ingles, C. J., Torregrosa, M. S., Ruíz-Esteban, C., Díaz-Herrero, A. y MartinezMonteagudo, M. C.(2010). Psychometric properties of the Academic Situations Specific Perceived Self-efficacy Scale in a Spanish students sample of Compulsory Secondary Education. European Journal of Education and Psychology, 3(1), 61-74. https://doi.org/10.30552/ejep.v3i1.51

García-Fernández, J.M., Vicent, M., Gonzávez, C. y Mañas, C. (2013). Attitudes towards Disability in Education through the SSCI (2000-2011): A Topical and Bibliometric Analysis. Electronic Journal of Research in Educational Psychology, 11(1), 139-166.

Genç, G. (2016). Attributions to Success and Failure in English Language Learning: The Effects of Gender, Age and Perceived Success. European Journal of Education Studies, 2(12), 26-43.

Gonzálvez, C., Giménez-Miralles, M., Vicent, M., Sanmartín, R., Quiles, M.J., \& García-Fernández, J.M. (2021). School Refusal Behaviour Profiles and Academic Self-Attributions in Language and Literature. Sustainability, 13, 7512. https://doi.org/10.3390/su13137512

Gonzálvez, C., Inglés, C. J., Kearney, C. A., Vicent, M., Sanmartín, R., \& García-Fernández, J. M. (2016). School Refusal Assessment Scale-Revised: Factorial invariance and latent means differences across gender and age in Spanish children. Frontiers in Psychology, 7, 2011. https://doi.org/10.3389/fpsyg.2016.02011

Gonzálvez, C., Sanmartín, R., Vicent, M., Inglés, C.J., Aparicio-Flores, M.P., \& García-Fernández, J.M. (2018). Academic self-attributions for success and failure in mathematics and school refusal. Psychology in the Schools, 55(4), 366-376. https://doi.org/10.1002/pits.22117

Guillen, J.C. (2020). Siete técnicas de estudio y aprendizaje efectivas. Cuadernos de pedagogía, (510), 121-126.

Kearney, C. A. (2002). Identifying the function of school refusal behavior: A revision of the School Refusal Assessment Scale. Journal of psychopathology and Behavioral Assessment, 24(4), 235-245. https://doi.org/10.1023/A:1020774932043

Kearney, C.A.; Gonzálvez, C.; Heyne, D. (2020). Editorial: School Attendance and Problematic School Absenteeism in Youth. Frontiers in Psychology. https://doi.org/10.3389/fpsyg.2020.602242 
Martinez-Monteagudo, M.C., Delgado, B.; Ingles, C.J. y García-Fernández, J.M (2019) Cyberbullying in the university setting. Relationship with family environment and emotional intelligence. Computers in Human Behavior, 91, 220-225. https://doi.org/10.1016/j.chb.2018.10.002

Marsh, H. W., Cairns, L., Relich, J., Barnes, J., \& Debus, R. L. (1984). The relationship between dimensions of self-attribution and dimensions of self-concept. Journal of Educational Psychology, 76(1), 3-32. https://doi.org/10.1037/0022-0663.76.1.3

Mendez, F.X., Espada, J.P., Orgiles, M., Hidalgo, M.D. y Garcia-Fernandez, J. M. (2008). Psychometric properties and diagnostic ability of the separation anxiety scale for children (SASC). European Child \& Adolescent Psychiatry, 17, 365-372. D0I: 10.1007/S00787-008-0678-8

Pérez, J.C.N., Pérez, C.R., Bernardo, A.B., \& García, D.Á. Problemas relacionados con el ámbito escolar. Consejo General de Colegios Oficiales de Psicólogos de España. Sanmartín, R., Vicent, M., Gonzálvez, C., GarcíaFernández, J. M., \& Inglés, C. J. (2016). Diferencias en las atribuciones académicas en Lengua en base al Afecto Positivo. International Journal of Developmental and Educational Psychology, 2(1), 143-149.

Ugarte, C. (2003). El derecho a la educación y la educación en derechos humanos en los textos internaciones de las naciones unidas y la Unesco. Pamplona: EUNSA.

Vega, L.E.G. (2021). La deserción escolar: Una problemática generalizada en las instituciones educativas. Sinopsis educativa. Revista venezolana de investigación, 21(1), 390-400.

Weiner, B. (1986). An Attributional Theory of Motivation and Emotion. Springer.

Weiner, B., \& Graham, S. (1990). Attribution in personality psychology. In L. A. Pervin (Ed.), Handbook of personality: Theory and research (pp. 465-485). The Guilford Press.

Weiner, B. (1992). Human Motivation: Metaphors, Theories, and Research. SAGE. 
